\title{
RETINO-CORTICAL INFORMATION TRANSMISSION ACHIEVABLE WITH A RETINA IMPLANT
}

\author{
Reinhard Eckhorn \\ Marcus Eger, Marcus Wilms, Thomas Schanze \\ Physics Deptm., Neurophysics Group, Philipps-University \\ Renthof 7, D-35032 Marburg, Germany \\ reinhard.eckhorn@physik.uni-marburg.de
}

\begin{abstract}
We are involved in the development of retina implants for blinds with photoreceptor degeneration. It has been shown by others that such blinds can perceive phosphenes elicited by retinal electrical stimulation. Key questions are: How should the stimulus information be coded in order to be sufficiently decodeable by the visual cortex and how much information can be transmitted with electrical stimulation to the visual centers in the spatial, temporal and amplitude domains? For ethical reasons we avoided explorative experiments with electrical retina stimulation in blind humans. Instead, we used the anesthetized cat and stimulated the retina focally alternating with visual stimuli and with sequences of short current impulses via an array of electrodes while we recorded intracortically in visual areas V1 and V2 with an array of microelectrodes [1]. Single- unit and multiple-unit spike activities were recorded in addition to local field potentials (LFP, 0.1 to 140 $\mathrm{Hz}$ ) in parallel by each of the 7 or 16 cortical electrodes. We used single (low rate) stimulation impulses and impulse trains with gamma interval distributions of different mean rates. For the various stimuli and recording signals we estimated the retino-cortical information transmission achievable with visual and electrical stimulation. We found information rates from single retinal stimulation- to single cortical recording-sites in the range of 20 to 140 bit per second, depending on the overlap between the receptive fields at the stimulation and recording locations and on the type of stimulation and the type of recorded signal. From these measures we estimated the temporal, spatial and contrast resolutions achievable with retina implants and compared them to those with visual inputs. The obtained values seem sufficient for visuo-motor coordination and object recognition in many in- and out-door situations of dayly life. However, the signals in visual cortex, evoked by visual and electrical stimulation, differ largely. It is therefore not clear in advance, how much and how fast the visual cortex can learn to decode the electrically evoked signals in order to make the implant a useful device for blind people.
\end{abstract}

Keywords: Shannon-information, electrical stimulation, population signals

\section{References}

[1] Schanze T, Wilms M, Eger M, Hesse L, Eckhorn R (2002) Activation zones in cat visual cortex evoked by electrical retina stimulation. Graefe's Archive Clin. Exptl. Ophthalmol. 240:947-954.

[2] Eger, M., Eckhorn, R. (2002) Assessing the encoding of stimulus attributes with rapid sequences of stimulus events. J. Comput. Neurosci. 13:207-216.

[3] Eger, M., Eckhorn, R. (2002) Quantification of sensory information transmission using timeseries decorrelation techniques. BioSystems 67:55-65.

[4] Eger, M., Eckhorn, R. (2002) A model-based approach for the analysis of neuronal information transmission in multi-input and -output systems. J. Comput. Neurosci. 12: 175-200. 